\title{
Kurt Seelmann
}

\section{Theologische Wurzeln des säkularen Naturrechts Das Beispiel Salamanca*}

Säkulares Naturrecht im Sinne eines Naturrechts, das sich nicht in erster Linie auf religiöse Begründungen und theologische Autoritäten beruft, hat man lange Zeit im 17. Jahrhundert, insbesondere mit Hugo Grotius, beginnen lassen ${ }^{1}$. In unserem Jahrhundert wird dann aber zunehmend die Kontinuität der Autoren des 17. Jahrhunderts mit der iberischen Spätscholastik, der „Schule von Salamanca“, des 16. Jahrhunderts betont ${ }^{2}$. Im folgenden soll weniger der Frage nachgegangen werden, von welchem Autor an sich sinnvoll von säkularem Naturrecht ${ }^{\text {" sprechen }}$ läßt, würde dies doch eine genauere Auseinandersetzung mit dem Begriff der Säkularisierung erfordern ${ }^{3}$, die hier nicht geleistet werden kann. Es soll aber an der iberischen Spätscholastik, von der unbestritten sein dürfte, daß sie ein wichtiges Moment in der Entwicklung zu einem säkularen Naturrecht darstellt, zweierlei gezeigt werden: wie naturrechtliche Argumentationen aus einem sehr freien und teilweise neuartigen Umgang mit theologischen Traditionen gewonnen werden und welche säkularen Problemstellungen sich dahinter verbergen.

In einem ersten Abschnitt soll gefragt werden, welche theologische Ausgangssituation sich für die Autoren des iberischen 16. Jahrhunderts in der Naturrechtsdebatte stellt (I). Exemplarisch soll dann der Umgang eines Juristen innerhalb der iberischen Spätscholastik mit dieser theologischen Tradition aufgegriffen werden (II). Am Schluß gilt ein kurzer Blick den praktischen sozialen Gegebenheiten und Bedürfnissen, die diese Diskussion leiten (III).

\footnotetext{
"Der Aufsatz greift einige Aspekte auf, die in meinen Schriften ${ }_{n}$ Die Lehre des Fernando Vázquez de Menchaca von dominium“ (Köln u.a. 1979) und „Theologie und Jurisprudenz an der Schwelle zur Moderne. Die Geburt des neuzeitlichen Naturrechts in der iberischen Spätscholastik" (Baden-Baden 1997) ausführlicher entwickelt sind.

1 Dazu Franco Todescan, Le radici teologiche del giusnaturalismo laico (Milano 1983) 9 f., im folgenden zitiert: Todescan.

2 Zur Literatur über die iberische Spätscholastik Christoph Bergfeld, Katholische Moraltheologie und Naturrechtslehre, in: Helmut Coing (Hrsg.), Handbuch der Quellen und Literatur der neueren europäischen Privatrechtsgeschichte (im folgenden zitiert: Coing, Handbuch) Band II 1 (München 1977) 1029-1033, im folgenden zitiert: Bergfeld.

3 Todescan, 1-9 gibt einen Problemüberblick.
} 


\section{Die theologische Ausgangssituation}

Römisches und kanonisches Recht verwendeten zwar den Begriff des Naturrechts, Grundfragen der menschlichen Existenz und des menschlichen Zusammenlebens waren aber im Verständnis des 16. Jahrhunderts weit mehr ein Gegenstand der Theologie, die für ihre Grundsatzüberlegungen nicht von ungefähr eine fast grenzenlose Zuständigkeit anderen Disziplinen gegenüber reklamierte. Francisco de Vitoria etwa hält fest: „Officium ac munus theologi tam late patet, ut nullum argumentum, nulla disputatio, nullus locus alienus videatur a theologica professione et institutione. ${ }^{4}$ Es waren insbesondere zwei theologische Traditionen, die im 16. Jahrhundert solche Fragen behandelten, die das säkulare Naturrecht übernehmen konnte: die Thomasrenaissance und der spätmittelalterliche Voluntarismus in der Tradition von Duns Scotus.

\section{Ein Lebrbuchwechsel}

1509 sind an der Universität von Paris die Sentenzen des Petrus Lombardus durch die in den 60er und 70er Jahren des 13. Jahrhunderts entstandene "Summa Theologiae" des Thomas von Aquin als den Unterricht beherrschendes Textbuch ersetzt worden ${ }^{5}$. Die Gründe dafür dürften, soweit wir das heute überblicken können, vielfältig gewesen sein. Die Sentenzen des Petrus Lombardus wurden als unzulänglich empfunden, weil sie lediglich Zitate aus der Väterliteratur aneinanderreihten und keinen selbständigen "roten Faden" theologischer Argumentation aufwiesen ${ }^{6}$. Klare Axiome aber schienen nun nötig, weil Lösungen für neue konkrete ethische und rechtliche Probleme deduziert werden sollten. Ein theologisches System mit ethischen Tugenden als Axiomen, wie es die IIa Ilae des Thomas anbot, mußte diesem Bedürfnis ganz besonders entgegenkommen. Auch die theoretische Nähe zu Aristoteles dürfte für Thomas gesprochen haben, da der Ideenrealismus die selbständige vernunftorientierte Lösung neuartiger ethischer Probleme eher begünstigte. Rein innertheologisch argumentierend hat man darin einen Sieg des Ideenrealismus sehen wollen?, was aber nur hinsichtlich der Konsequenzen der Entwicklung teilweise richtig ist. Mindestens so bedeutsam für diesen Wechsel dürfte die Überlegung gewesen sein, daß die juristisch und ethisch unergiebigen Sentenzen des Petrus Lombardus den Differenzierungsbedürfnissen

4 Francisco de Vitoria, Über die staatliche Gewalt. De Potestate Civili. Eingeleitet und übersetzt von Robert Schnepf (Berlin 1992) 32.

5 Bergfeld, 1016.

${ }^{6}$ Dazu mit Nachweisen Frank Grunert, Theologien der Strafe. Zur Straftheorie von Thomas von Aquin und ihrer Rezeption in der spanischen Spätscholastik: das Beispiel Francisco de Vitoria, in: Hans Schlosser, Dietmar Willoweit, Neue Wege strafrechtsgeschichtlicher Forschung (Köln 1999) 313, 316; im folgenden zitiert: Grunert.

7 So Hans Welzel, Naturrecht und materiale Gerechtigkeit, 2. unveränd. Nachdruck der 4. Aufl. (Göttingen 1990) 90, 91, im folgenden zitiert: Welzel. 
des forum internum einfach nicht mehr genügten ${ }^{8}$ und daß der Stil von Thomas stärker der nun nach der Erfindung des Buchdrucks entstehenden Handbuchmentalität ${ }^{9}$ entgegenkam. Diese Auswechselung des Unterrichtsmaterials fand sehr schnell Verbreitung - unter anderen haben die Theologen der Schule von Salamanca diese Entscheidung übernommen. Bei den nunmehr entstehenden Thomas-Kommentaren fällt auf, daß sie, darin die großen Beichtsummen ablösend, sich ganz zentral mit den moraltheologisch relevanten Teilen der „Summa Theologiae“ befassen. Eine Reihe von Autoren wie Vitoria, Soto, Molina und andere lesen darüber und veröffentlichen zum Teil mehrbändige Kommentare ${ }^{10}$.

Dieser für das Naturrecht relevante Teil der „Summa Theologiae“ teilt in der Tat mit der gesamten thomistischen Theologie deren ideenrealistische Ausrichtung. Der Ideenrealismus stellt, vereinfacht gesagt, die göttliche Vernunft über den göttlichen Willen, bindet gewissermaßen Gott selbst an seine lex naturalis. Durch die Abtrennung dieses Naturrechts von einem es potentiell modifizierenden göttlichen Willen wird eben dieses Naturrecht als durch Vernunft für Menschen erkennbar und begründbar behauptet ${ }^{11}$. Diese Sicht der Dinge, zusammen mit dem Umstand, daß es sich um die Kommentierung eines theologischen Werkes handelte, gab den Kommentatoren eine große Freiheit gegenüber den ihnen gleichwohl bekannten juristischen Autoritäten und Texten. Was diese Freiheit für Juristen bedeutete, wird gleich noch aufzugreifen sein.

\section{Die voluntaristische Trennung von Faktum und Norm}

Zunächst aber sei noch auf die zweite das 16. Jahrhundert bestimmende theologische Traditionslinie hingewiesen. Keineswegs nämlich war die iberische Spätscholastik ausschließlich von einer Thomasrenaissance geprägt. Auf einige Vorsicht hätten sicher schon die Umstände hinweisen müssen, daß Vitoria immerhin im voluntaristischen Paris studiert hatte und daß es auch in der Blütezeit der iberischen Spätscholastik sogar in ihrem intellektuellen Zentrum, in Salamanca, auch scotistische Lehrstühle gegeben hat ${ }^{12}$. Es kann keineswegs von einem - gar noch "endgültigen “ - Sieg des Thomismus über den Scotismus, des Ideenrealismus über den Voluntarismus ${ }^{13}$ gesprochen werden. Es gibt nicht einfach die nantinominalistische spanische Spätscholastik“14. Vielmehr liegt eine ziemlich komplizierte Entwicklung auf der Basis mehrerer Traditionslinien vor, die im übrigen allein aus

8 Daniel Deckers, Gerechtigkeit und Recht. Eine historisch-kritische Untersuchung der Gerechtigkeitslehre des Francisco de Vitoria (1483-1546) (Freiburg i.Ue., Freiburg i.Br. 1991) 19, im folgenden zitiert: Deckers.

${ }^{9}$ Niklas Lubmann, Subjektive Rechte. Zum Umbau des Rechtsbewußtseins in der modernen Gesellschaft, in: ders., Gesellschaftsstruktur und Semantik, Bd. 2 (Frankfurt a.M. 1981) $45 \mathrm{ff} ., 49$, im folgenden zitiert: Lubmann.

10 Aufgelistet bei Bergfeld, 1025, $1026 \mathrm{f}$.

11 Welzel, $89 \mathrm{ff}$., $95 \mathrm{ff}$.

12 Bergfeld, 1016.

13 So Bergfeld, 1016, relativiert 1019.

14 So Welzel, 91. 
dem Thomismus heraus überhaupt nicht erklärbar wäre. Das ergibt sich nicht nur daraus, daß die zentrale Aussage des Voluntarismus, daß nämlich Gott, wenn er gewollt hätte, die Welt ganz anders hätte ordnen können, auch in der iberischen Spätscholastik begegnet ${ }^{15}$. Es wird auch nur unzureichend dadurch charakterisiert, daß die Vorstellung vom Recht als Kunst zunehmend der vom Recht als corpus von Geboten und subjektiven Rechten weiche, daß, wie Villey provokativ überzeichnet, ars durch thora abgelöst wird ${ }^{16}$. Wichtig ist vielmehr, daß Duns Scotus Grundzüge eines Naturrechts zu entwickeln versucht hat, das nicht mehr auf der Idee einer vernunftgemäßen Ableitung aus obersten Prinzipien, sondern vielmehr auf Gottes Willen beruhen sollte ${ }^{17}$. Nicht ein Naturzustand mit der ihm immanenten Teleologie, sondern eine vom bloß Faktischen getrennte Norm sollte einem solchen Naturrecht zugrunde liegen. Hintergrund dafür ist die Demonstration, daß nicht einmal die Gebote der zweiten Tafel des Dekalogs im strengen Sinn aus dem Gebot der Gottesliebe deduziert werden können ${ }^{18}$. Das Naturrecht erfährt so durch Duns Scotus und seine Tradition eine Subjektivierung und Norma tivierung.

\section{Der Umgang der Juristen mit der theologischen Tradition}

Auf dieser Basis ist nunmehr zu fragen, wie Juristen in dieser Zeit mit dem praktischen Bedürfnis nach einem säkularen Naturrecht und mit den theologischen Traditionen umgehen konnten. Beispielhaft soll dies an Fernando Vázquez, dem einzigen bekannten Legisten innerhalb der iberischen Spätscholastik, gezeigt werden. Als Nichttheologe und Nicht-Kirchenrechtler verkörpert er am ehesten den Typ eines frühen säkularen Naturrechts.

\section{Thomistisches}

Wie nimmt Vázquez die Thomasrenaissance auf? Vázquez sucht die Schwelle staatlicher Enteignungen von Rechtspositionen der Bürger zu erhöhen und sie nicht mehr nur von bestimmten formellen Voraussetzungen abhängig zu machen,

15 Kurt Seelmann, Die Lehre des Fernando Vázquez de Menchaca von dominium (Köln u.a. 1979) 24, im folgenden zitiert: Seelmann, Vázquez.

${ }^{16}$ Michel Villey, La promotion de la loi et du droit subjectiv dans la seconde scolastique, in: Paolo Grossi (Hrsg.), La seconda scolastica nella formazione del diritto privato moderno (Milano 1973) $53 \mathrm{ff}, 60 \mathrm{ff}$.

17 Johannes Duns Scotus, Quaestiones in Lib. I-IV Sententiarum, in: Opera Omnia vol. V-X (Darmstadt 1968), In: III Sent., dist. 19, q. unica, schol., nu. 7, im folgenden zitiert: Duns Scotus.

${ }_{18}$ Dazu Karl-Heinz Ilting, Stichwort „Naturrecht“ in: Geschichtliche Grundbegriffe. Historisches Lexikon zur politisch-sozialen Sprache in Deutschland 4, hrsg. von Otto Brunner, Werner Conze, Reinhart Koselleck (Stuttgart 1978) $266 \mathrm{ff}$. 
sondern davon, daß sie für das Gemeinwohl unerläßlich sind ${ }^{19}$. Zur Stärkung dieser Argumentation muß er zum einen den Begriff des dominium, in das schon nach bisheriger Lehre immerhin nur ex causa eingegriffen werden konnte, mit Hilfe einer Weiterentwicklung einer legistischen Tradition auf alle (subjektiven) Rechtspositionen ausdehnen ${ }^{20}$. Zum anderen will er die mit dem Innehaben des dominium verbundene Rechtsposition dadurch stärken, daß er sie mit dem Freiheitsbegriff verbindet. Dies gelingt mit Hilfe einer Anknüpfung an Soto und die thomistische Tradition. Soto verknüpft das (für Vázquez relevante) dominium externarum rerum dadurch mit dem dominium suorum actuum, daß er letzteres zur Voraussetzung für ersteres erklärt: „natura ipsa nemo est dominus rerum nisi est dominus actionum suarum" 21 . Er bezieht sich dafür auf die Prima Secundae des Aquinaten, wo allerdings das dominium suorum actuum noch nicht als Voraussetzung des dominium externarum rerum auftaucht, sondern im Kontext mit dem theologischen Problem des liberum arbitrium ${ }^{22}$, das er, zuerst wohl im Sentenzenkommentar, als dominium sui actus umschreibt ${ }^{23}$. Das Beispiel mag zugleich zeigen, daß die Bezugnahme auf Thomas nicht selten einen erheblichen Argumentationsaufwand erforderte und ihrerseits bei den Theologen der Zeit schon eine ziemliche Selbständigkeit im Umgang mit den Traditionen dokumentiert.

Man kann diese Selbständigkeit in einem sehr engen Zusammenhang mit dem angesprochenen zunehmenden Gewicht individueller Rechtspositionen auch bei einem anderen Autor, Francisco de Vitoria, aufzeigen: In seinem Thomaskommentar stimmt er zunächst Thomas darin zu, daß es lobenswert sei, auf rechtliche Ansprüche Verzicht zu leisten und persönliches Unrecht geduldig hinzunehmen, um dann aber deutlich hinzuzufügen, daß rechtliche Genugtuung (satisfactio) zu fordern weder ungerecht noch unerlaubt sei24.

\section{Divisio rerum: Zustand, Erlaubnis oder Gebot?}

Nimmt man die scotistische Tradition noch hinzu, so zeigt sich das Rechtsverständnis der iberischen Spätscholastik beim Blick auf die Quellen gerade im Detail als eine durchaus originelle Kombination aus aquinatischem Vernunftoptimismus und scotistischer Normativität. Machen wir uns diesen Zusammenhang an einem für die Diskussion des 16. Jahrhunderts zentralen Thema deutlich: an der Frage, wie das seit Isidor von Sevilla ${ }^{25}$ in der gesamten mittelalterlichen Tradition selbst-

${ }^{19}$ Fernando Vázquez, Controversiarum Illustrium aliarumque usu frequentium libri tres (Venedig 1564) lib. I, cap. 17, nu. $1 \mathrm{ff}$. , im folgenden zitiert: Vázquez.

20 Vázquez, lib. I, cap. 17, nu. $4 \mathrm{ff}$.

21 Francisco de Soto, Libri decem de iustitia et iure (Lyon 1569) lib. IV, q. 1, a. 2, im folgenden zitiert: Soto.

22 Thomas von Aquin, Ia, Ilae, q. 1, a. 1.

23 Thomas von Aquin, Commentum in lib. II Sententianum, d. 25, q. 1, a. 1.

${ }^{24}$ Francisco de Vitoria, Comentario a la secunda secundae de Santo Tomás. Edición Vicente Beltrán de Heredia, tom. I (Salamanca 1932) q. 108, 1, n. 4. Dazu Grunert, 330.

25 Isidor von Sevilla, Etymologiarum sive originum libri XX (Oxford 1911) V 4, 1; im folgenden zitiert: Isidor. 
verständlich vorausgesetzte ursprüngliche omnia communia später doch zu privatem Recht an Land werden, am Meer aber bestehen bleiben konnte. Hierzu sei kurz ein Blick auf die drei bedeutendsten Autoren des spanischen 16. Jahrhunderts, Vitoria, Soto und wieder Fernando Vázquez, geworfen. Für sie alle gilt als Voraussetzung das von Gratian in einem Introductorium in sein Dekret übernommene omnia communia als ein ius naturae ${ }^{26}$. Sie fragen, wie daraus trotzdem eine divisio rerum entstehen konnte. Thomas von Aquin, auf den die Autoren des 16. Jahrhunderts natürlich eingehen, hat das Problem dadurch gelöst, daß er das omnia communia zu einem bloßen Naturzustand erklärt hat, der durch menschliches Recht aus Gründen der Zweckmäßigkeit jederzeit geändert werden konnte: „Ad tertium dicendum quod aliquid dicitur esse de iure naturali dupliciter. Uno modo, quia ad hoc natura inclinat: sicut non esse iniuriam alteri faciendum. Alio modo, quia natura non induxit contrarium: sicut possemus dicere quod hominem esse nudum est de iure naturali, quia natura non dedit ei vestitum, sed ars adinvenit. Et hoc modo communis omnium possessio... dicitur esse de iure naturali. “27

Für alle drei spätscholastischen Autoren spitzt sich das Problem nun dadurch zu, daß sie das omnia communia, ohne sich diesen Unterschied überhaupt klar zu machen, im Gegensatz zu Thomas und mit Duns Scotus und Ockham normativ verstehen. Vitoria und Soto sprechen von einer göttlichen Erlaubnis: „Sed illud nunquam fuit praeceptum, sed concessum est ut omnia essent communia “ konstatiert Vitoria ${ }^{28}$, und Soto schließt sich an: „Sed eo sensu negativo dicitur communis possessio de iure naturae, quod nunquam lex naturalis eandem praecepit divisionem, sed permisit hoc vel illo modo possideri." 29 Vázquez geht gar von einem göttlichen Gebot aus, wie sich darin zeigt, daß er am dominium in communi eine praescriptio aus lange andauernder Übung gegen das ius gentium primaevum für unzulässig erklärt: „Est contra ipsum ius naturae, aut gentium primaevum, quod mutari non posse diximus. " 30 Dennoch gehen aber alle drei Autoren nicht soweit wie Duns Scotus, der eine ausdrückliche göttliche revocatio für die dann durch Menschen vorgenommene Aufteilung des omnia communia postuliert und mit dem menschlichen Sündenfall begründet hatte: ${ }_{n} . .$. conclusio est, quod illud praeceptum legis naturae, de habendo omnia communia, revocatum est post lapsum... "31. Vitoria und Soto können so immerhin wie Thomas trotz ihrer normativen Aufladung des omnia communia dessen Außerkraftsetzung durch menschliches Recht behaupten. Schwieriger wird die Situation für den Juristen Vázquez, der sich deshalb sogar noch intensiver auf den theologischen Diskurs einlassen muß als seine beiden theologischen Zeitgenossen in Salamanca. Er hat immerhin

26 Gratian, Introductorium zu D. 8 c. 1: „Nam iure naturae sunt omnia communia omnibus ...".

27 Thomas von Aquin, IIa, Ilae, q. 66, a. 1.

28 Francisco de Vitoria, De iustitia, Ed. Beltran Beltrán de Heredia, tom. 1 (Madrid 1934) q. 62 , a. 1 , nu. 20, im folgenden zitiert: Vitoria, De iustitia.

29 Soto, lib. IV, q. 3, a. 1 .

30 Vázquez, lib. II, cap. 89, nu. 30.

${ }^{31}$ Duns Scotus, In IV Sent., dist. 15, q. 2, nu. 5. 
von Duns Scotus den Gebotscharakter des omnia communia übernommen, ist aber doch, beeinflußt durch Thomas, der rein voluntaristischen Lösung des Problems gegenüber zu skeptisch. So kommt für Vázquez keine revocatio des Gebots in Betracht, sondern nur eine so vorher nicht vorhandene komplizierte Prinzipienabwägung 32: Weil das Unterlassen einer Aufteilung am Land unter anderem zu Hungersnöten führen würde, dies aber noch eindringlicher Gottes Geboten widersprechen würde, dürften in Befolgung des noch wichtigeren göttlichen Gebots die Menschen das Gemeineigentum aufteilen: „Constat enim, quod si multi venentur, aut piscentur in terra, vel flumine, facile nemus feris, et flumen piscibus evacuatum redditur. ${ }^{33}$ Neben ihrer originellen Verbindung zweier theologischer Traditionslinien zeigt diese Argumentation im übrigen auch, wie frei Juristen mit ihrer eigentlich juristischen Tradition umgehen: Für die legistische Tradition, die das kanonistische omnia communia nie ganz übergehen konnte, war die divisio rerum schon sehr früh, gewissermaßen in einem produktiven Mißverständnis, zur feudalistischen Aufteilung in Obereigentum und Untereigentum mutiert ${ }^{34}$. Vázquez sieht das Mißverständnis und wendet sich konsequent der entscheidenden theologischen Dimension des Problems zu.

Beachtung verdient bei alledem noch eine weitere Paradoxie: Die eher naturrechtsskeptische scotistische Tradition, in der auch die schon genannten Franziskaner mit ihrer dem Recht gegenüber generell skeptischen Armutsforderung standen, hat mit ihrer klaren Postulierung der Normativität des Rechts eine der Vorbedingungen der späteren Systematisierung des Naturrechts und seiner endgültigen Verabschiedung eines teleologischen Naturverständnisses geschaffen.

\section{Gespaltenes Naturrecht und Korrektur von Naturrecht}

Betrachten wir schließlich, wie Vázquez aus den verschiedenen Traditionslinien unter Einschluß der Theologie einen für das säkulare Naturrecht weiterführenden gespaltenen Naturrechtsbegriff entwickelt.

Zwar kennt auch die Tradition vor dem 16. Jahrhundert unterschiedliche Begriffe von Naturrecht, wobei dies insbesondere mit unterschiedlichen Definitionen im justinianischen und im kanonischen Recht zusammenhängt ${ }^{35}$. Gleichwohl betreffen die früheren Differenzen im wesentlichen die Frage, ob unter Naturrecht das allen Lebewesen gemeinsame Recht zu begreifen sei - der Begriff der Natur wäre dann im wesentlichen identisch mit unserem heutigen biosoziologischen Naturbegriff - oder aber, ob man unter Naturrecht nur die für Menschen

32 Vázquez, lib. II, cap. 89, nu. 39 i. Verb. mit lib. I, cap. 9, nu. 4.

33 Vázquez, lib. II, cap. 89, nu. 39.

34 z.B. Bartolus, Anm. 4 zu D. 1.1.5. unter dem für einen Legisten naheliegenden Hinweis darauf, daß die andere (ihm bekannte) Interpretation in Widerspruch geriete zu der Stelle Inst. 4.1.1., wo der Diebstahl als naturrechtlich verboten angeführt wird.

35 D. 1.1.3., Inst. 1.2 princ.: „ius naturale “ als "quod natura omnia animalia docuit “; dagegen D. 1 c. 7: nius naturale est commune omnium nationum" (nach Isidor V 4, 1) und D. 1 c. 1: nius naturale est, quod in lege et evangelio continetur ${ }^{*}$. 
geltenden Vernunft-Gebote außerhalb des Bereichs der christlichen Offenbarung im engeren Sinne verstehen solle ${ }^{36}$. Das so gut wie allen Menschen gemeinsame aber gleichwohl von Menschen geschaffene Recht gehörte unter den Begriff des ius gentium zum menschlichen Recht und jedenfalls im Prinzip nicht zum Naturrecht $^{37}$. Über diese Entscheidung legt sich nun freilich schon im justinianischen Recht eine andere, die $\mathbf{z w i s c h e n} \mathrm{zweierlei}$ allein den Menschen betreffenden iura naturalia oder iura gentium differenziert. Das Problem entzündet sich an der natürlichen Verpflichtung des Sklaven ${ }^{38}$, der entsprechend den legistischen Quellen nach ius gentium unfrei, nach eben demselben ius gentium aber durch eine Naturalobligation verpflichtet sein konnte. Man behalf sich mit einer $Z$ weiteilung des ius gentium: nach dem ursprünglichen und allen Menschen gemeinsamen Recht könne auch der Sklave natürlich verpflichtet werden, erst durch ius gentium secundarium habe er dann seine Freiheit verloren ${ }^{39}$. Auch hier stehen also die Juristen des 16. Jahrhunderts, die eine ähnliche Aufteilung aufgreifen, durchaus innerhalb der juristischen Tradition. Dennoch gewinnt die Differenz nun im 16. Jahrhundert eine neue Bedeutung. Im Fall der obligatio naturalis ging es nicht unmittelbar darum, daß eine Regelung des erstrangigen ius gentium durch eine des zweitrangigen ius gentium verdrängt wird; man versuchte nur zu erklären, warum bei einigen Menschen (den freien Bürgern) zu dieser naturalis obligatio einklagbare Verpflichtungen hinzukommen, bei den anderen (den Sklaven) dies aber nicht der Fall ist. Insofern ergänzen in der Tradition die beiden iura gentium einander, während man im 16. Jahrhundert dann die spätere Sklaverei nach der ursprünglichen Freiheit für erklärungsbedürftig hält. Eine solche Erklärung für das 16. Jahrhundert konnte sein, daß die Sklaverei eingeführt wurde, um einen noch größeren Nachteil zu verhindern (maioris malis vitandi gratia), nämlich die Tötung des besiegten Feindes. Eine Abwägung zwischen Freiheit und Leben führt dann zur Legitimation der kriegsbedingten Sklaverei ${ }^{40}$.

In der iberischen Spätscholastik wird nun aber dieses Doppelschema der iura gentium oder iura naturalia (Begriffe, die nunmehr austauschbar werden), eines von Anfang an vorhandenen und eines dem zeitlichen Wandel unterworfenen, zusätzlich auf einen Bereich übertragen, innerhalb dessen seine alte Hierarchie nicht mehr paßt. Es soll nun nämlich, wie zu sehen war, auf den Bereich der Aufhebung des omnia communia Anwendung finden. Angesichts des normativen Charakters des omnia communia muß für die es aufhebende divisio rerum eine Rechtsnorm von gleicher Dignität geschaffen werden, die gleichwohl nicht derselben Geltungsebene angehören kann. Auch dies aber wird im Salamanca des 16. Jahrhunderts aus der weitergedachten Sklavenproblematik entwickelt, indem auch bei der

36 Ausführlich Seelmann, $108 \mathrm{ff}$.

${ }^{37}$ D. 1.1.1.4.: nius gentium est, quo gentes humanae utuntur". Vgl. zu Gratian D. 1 c. 9.

38 Dazu Paulus de Castro, In primam digesti veteris partem commentaria (Venedig 1593) Anm. princ. zu D. 1.1.5.

39 Jason de Mayno, In primam digesti veteris... commentaria (Venedig 1573) Anm. 1 zu D. 12.6.64.

40 Vázquez, lib. I, cap. 9, nu. 4. 
divisio rerum die Abwägung, in diesem Fall zugunsten des Überlebens der Menschheit, vorrangiges späteres Naturrecht schafft, das nun sogar Gebote des ursprünglichen Naturrechts außer Kraft setzen kann. So entwickelt sich im Falle von Vázquez bei einem \uristen mit Hilfe der schon genannten moraltheologischen Argumentation eine Begründung dafür, warum das Innehaben von Rechten nach dem späteren Naturrecht anders geregelt wird als nach dem ursprünglichen Naturrecht.

Auch dies Außerkraftsetzen von normativem Naturrecht ist nichts völlig Neues. Bereits Gratian behandelt in der distinctio 13 des Dekrets die Frage, ob gegen ius naturale eine dispensatio zulässig sei, ob also die kirchliche Autorität im Einzelfall das Abweichen von einem ius naturale einräumen dürfe ${ }^{41}$. Dies wird zwar grundsätzlich bestritten, in Ausnahmefällen aber zugelassen, nämlich dann, wenn es zwischen zwei Übeln zu wählen gelte. Als Schulbeispiel hierfür galt in der dekretistischen Literatur der Schwur, jemanden zu töten ${ }^{42}$. In einer solchen Situation müsse man das größere Übel, das Töten, vermeiden, dafür aber das geringere Übel verwirklichen, nämlich den Schwur brechen. Freilich ist diese Argumentation in der Kanonistik zur Lösung eines Einzelfalls entwickelt worden, während sie im 16. Jahrhundert das allgemeine Verhältnis zweier Naturrechtsarten bestimmen soll.

Auch hier sind es so wieder zwei Traditionslinien, die in der iberischen Spätscholastik etwas Neues entstehen lassen. Aus der Verdoppelung eines die Menschen betreffenden Naturrechts im speziellen Fall der Naturalobligation des Sklaven in der legistischen Literatur einerseits und der kanonistischen Lehre, wonach im Einzelfall eine kirchliche dispensatio vom Naturrecht zulässig sein könne, wird mit Hilfe zweier moraltheologischer Konstruktionen zur Begründung der divisio rerum (der thomistischen und der scotistischen) ein im Ergebnis doch gänzlich neues System mit zwei Naturrechtsebenen entwickelt. Diese Konstruktion gibt den Autoren einen gewaltigen Spielraum nicht nur gegenüber positivem römischen oder kanonischen Recht oder dem Statutarrecht, sondern sogar gegenüber dem in der Tradition für unverbrüchlich gehaltenen Naturrecht erster Ordnung. Wenn diese Lehre gerade beim dominium entwickelt wurde, mag dies damit zusammenhängen, daß in dieser Zeit die Vorstellung einer bloß positiv-rechtlichen Korrektur des omnia communia an Plausibilität verliert, da "mehr und mehr Eigentum auf Arbeit und/oder vertraglichem Erwerb zurückgeht ${ }^{433}$.

Um die Sprengkraft und wirkungsgeschichtliche Bedeutung einer solchen Konstruktion einschätzen zu können, bedarf ein bisher vernachlässigter Aspekt noch der Betrachtung. Unabhängig davon, ob man für die divisio rerum scotistisch eine revocatio voraussetzte oder aber in der thomistischen Tradition dies nicht verlangte, so war doch, selbst nach einer revocatio, noch ein menschliches Handeln

41 D. 13 c. 1.

42 Rufinus, Summa Decretorum, Neudruck der Ausgabe Paderborn 1902 (Aachen 1963), Anm. zu D. 13 c. 1; Summa Parisiensis, Ed. McLaughlin (Toronto 1952) Anm. zu D. 13 c. 1. 43 Lubmann, 70. 
nötig44. Von Scotus bis Vitoria werden hierfür drei mögliche Wege genannt: Entweder der erste Mensch (oder Noe) hat mit patria potestas diese Teilung vorgenommen, oder die Menschen haben zu diesem $Z$ weck einen Herrscher eingesetzt oder aber die Teilung geschah unmittelbar durch Konsens: Soto faßt zusammen: nAd hoc dicendum... quod tripliciter potuit hoc fieri: Primo quod Adam habuerit de se potestatem ad dividendum, non quod talis sit potestas paterna universaliter, sed quod esset singularis in Adam quia erat principium naturae sicut habuit alias praerogativas super alios patres. Sed tamen, quia non videtur fundari in Sacra Scriptura quod haberet talem vim coactivam, melius est dicere secundo modo: $V_{1}$ detur quod hoc fuit ex consensu omnium, videlicet quod consenserunt omnes ut ipse divideret illis terram, vel elegerunt alium superiorem. Vel, tertio, fuit inter eos conditum ad dirimendos quaestiones ut unus acciperet unam partem, alius aliam..." ${ }^{45}$. Konsens ließ sich dabei, wie Vitoria zeigt, auch als stillschweigender Konsens oder gar als bloße Gewöhnung verstehen: ${ }_{n}$ Et forte sic facta fuit, non consensu certo et formali, sed quodam consensu interpretativo, ita quod incoeperint aliqui colere certas terras et alius alias. ${ }^{~}{ }^{46}$ Kombiniert man solche Einigungstheorien dann, wie bei Vázquez, mit einem zweifach geschichteten Naturrecht, dessen zweite, zeitlich spätere Schicht, das ursprüngliche Naturrecht sogar als Ergebnis von Abwägungen abändern kann, so ist der Weg ersichtlich nicht mehr weit bis zu Suarez' Entwicklung eines pactum unionis und eines pactum subiectionis Ende des 16. Jahrhunderts ${ }^{47}$ und bis zu den dann gar nicht mehr so revolutionären Gesellschaftsvertragskonstruktionen zur Begründung des Naturrechts im 17. Jahrhundert.

\section{Die säkularen Problemstellungen}

Der theologische Streitstand sowie seine selbständige Verwendung in der juristischen Naturrechtslehre von Vázquez sind bisher nur auf der theoretischen Diskussionsebene vorgeführt worden. Gleichwohl war immer schon der Hintergrund säkularer Problemstellungen spürbar, um deren Lösung es bei den theologischen Bemühungen ging. Diese seien abschließend noch einmal ausdrücklich erwähnt - vorab ist freilich auch die staatspolitische Situation noch zu benennen, die es Vázguez als Juristen ermöglicht, vergleichsweise selbständig auch mit der juristischen Tradition umzugehen.

44 Duns Scotus, In III Sent., dist. 19, q unica, schol., nu. 6.

45 Soto, Relección nde dominio“. Ed. Jaime Brufau Prats (Granada 1964), 118.

46 Franciso de Vitoria, De iustitia, nu. 23.

47 Francisco Suarez, Tractatus de legibus et legislatore Deo (Coimbra 1612) lib. III, cap. 4, nu. $1 \mathrm{f}$. 


\section{Die staatspolitische Situation im Salamanca des 16. Jabrbunderts}

Es fällt auf, daß Vázquez dem römischen Recht durchaus mit einigem Abstand begegnet, ja die theologische Tradition der römischrechtlichen bei der Problemlösung mitunter vorzieht. Dahinter steht nicht nur persönliche Unabhängigkeit, sondern auch die staatspolitische Situation Spaniens. Da die iberische Halbinsel nicht Reichsgebiet war, hatte sich dort auch nicht die Vorstellung einer direkten oder auch nur subsidiären Geltung des römischen Rechts ausgebildet ${ }^{48}$. Gesetzgebung und Rechtswissenschaft hatten so von vornherein einen großen Spielraum beim Umgang mit Rechtsquellen, was als "souveräner Synkretismus" ${ }^{49}$ oder "quellensynkretistische Behandlung " 50 bezeichnet worden ist. Das römische Recht wurde zwar an den Universitäten gelehrt. Ziel war aber fast ausschließlich die Methodenorientierung der Juristenausbildung und eine Gliederung des Rechtsstoffes. Deshalb ist auch eine anwendungsbezogene Kommentarliteratur zum römischen Recht in Spanien wenig verbreitet ${ }^{51}$. Das aber machte wiederum westgotisches Volksrecht, königliches Recht und Stadtrechte anhand eines anspruchsvollen Maßstabs kritikabel. Aus dem Synkretismus entstand ein gewisses Wahlrecht bei der Suche nach Kollisionsregeln, die sich den genannten Rechtsmaterien nicht entnehmen ließen. Zwar hatte z. B. für Kastilien das Ordenamiento de Alcalá 1348 eine Rangfolge der Rechtsquellen festgelegt ${ }^{52}$, die aber immer umstritten blieb und angesichts der mehrdeutigen Funktion der "Siete Partidas " und der Lückenhaftigkeit vorrangiger Quellen leicht umgangen werden konnte ${ }^{53}$. Anders als im Bereich des unmittelbaren Geltungsanspruchs des römischen Rechts war die juristische Aufgabe deshalb von vornherein weniger die einer Harmonisierung der (als nur scheinbar ausgewiesenen) Widersprüche in den Quellen. Vielmehr scheint bereits diese Tradition eine offen demonstrierte Suche nach naturrechtlich begründeten besseren Lösungen, auch aus dem Bereich der Theologie, geradezu begünstigt zu haben.

\section{Der aktuelle Bedarf nach Naturrecht}

Neben dieser dem Entstehen auch theologisch begründeten Naturrechts förderlichen staatspolitischen Ausgangslage bedürfen noch zwei aktuelle Problemfelder

48 Legistische Literatur findet sich generell in Spanien nur spärlich, vgl. Norbert Horn, Die legistische Literatur der Kommentatoren. Länderbericht Spanien, in: Coing, Handbuch Bd. I (München 1973) $294 \mathrm{ff}$, im folgenden zitiert: Hom.

49 Hans Thieme, Natürliches Privatrecht und Spätscholastik, in: ZRG GA 70 (1953) $230 \mathrm{ff}$, 264.

50 Michael Holthöfer, Literatur in Italien, Frankreich, Spanien und Portugal, in: Coing, Handbuch Bd. II 1, 242.

51 Hom, 295.

52 Armin Wolf, Die Gesetzgebung der entstehenden Territorialstaaten, in: Coing, Handbuch Bd. I, 673 .

53 Maria Scheppach, Las Siete Partidas. Entstehung und Wirkungsgeschichte (Pfaffenweiler 1991) 49. 
der Erwähnung, die schon in dem kurzen Überblick über die naturrechtliche Argumentation von Vázquez durchscheinen: Die Eroberung Amerikas als Begegnung mit fremden Kulturen und die sich wandelnde Wirtschaft erfordern in der Sicht der Zeit offensichtlich eine Kolonialethik und eine neue Wirtschaftsethik.

Die Entdeckung Amerikas führte zur Begegnung mit bisher fremden Kulturen. Aus dieser einseitig - von den europäischen Mächten - dominierten Begegnung entstanden sehr bald auch viele juristische Fragen. Sie betrafen insbesondere die Rechte, die die europäischen Eroberer im Umgang mit den Eroberten hatten. Es war nicht einmal selbstverständlich, daß diese fremden Wesen überhaupt rechtsfähig waren - ihnen die prinzipielle Rechtsfähigkeit zuzuerkennen war bereits Ergebnis einer langen Diskussion insbesondere am spanischen $\mathrm{Hof}^{54}$. Hatten diese Menschen aber im Einzelfall auch ein Lebensrecht und ein Freiheitsrecht, hatten sie gar ein Eigentumsrecht? Die klassische Sklaverei- Problematik und ihre Argumentationsfiguren tauchten auf - wie auch bei Vázquez zu sehen war - sie gab aber keine zufriedenstellenden Antworten, wollte man nicht alle Bewohner der eroberten Gebiete als Kriegsgefangene behandeln. Aber auch die traditionelle dominium-Problematik war neu zu überarbeiten - für Vázquez ein zentrales Anliegen. Die klassische Abhandlung zur Stellung der Eroberten stammt von Vitoria, der den Eroberten zwar ein Eigentumsrecht zuspricht, sie aber wie Kinder unter die Vormundschaft der spanischen Krone stellen will ${ }^{55}$. Römisches und kanonisches Recht gaben für solche Fragenkreise keine eindeutigen Antworten. Die bisherige Naturrechtslehre war zwar Argumentationsgrundlage, aber legte die Ergebnisse gleichfalls nicht fest. Ius naturale war vordem kein Begriff, aus dem man durch Deduktion die Entwicklung konkreter Rechtssätze für bisher unbekannte Problembereiche hätte ermöglichen können. Die praktische Bedeutung des Naturrechts war eher negativ: Man brauchte in der Beichtjurisprudenz Kriterien dafür, wann ein Gesetz gegen Naturrecht verstieß und also nicht in conscientia verpflichtete mit der Folge, daß der Verstoß dagegen keine Sünde war ${ }^{56}$. Nun aber mußten naturrechtliche Argumente die Grundlage dafür hergeben, daß man positives Recht für den Umgang mit den Indianern schaffen konnte ${ }^{57}$.

Ein zweiter Grund für den aktuellen Bedarf an Naturrecht lag - auch dies zeigt das Beispiel von Vázquez - im Bereich der in einer Umbruchsituation befindlichen Wirtschaft. Neben dem immer wieder erwähnten Bedürfnis nach Vertragsund Formfreiheit und damit einer Versprechenslehre, einer Willensethik und einer

54 Dazu Antonio-Enrique Pérez Luño. Die klassische spanische Naturrechtslehre in 5 Jahrhunderten (Berlin 1994) 28, 185; Rainer Specht, Darf der König von Spanien Indianer unterwerfen?, in: Nation-Nationalstaat-Nationalismus, Rechtsphil. Hefte III (1994) 51 ff, 56.

55 Francisco de Vitoria, De indis recenter inventis et de iure belli Hispanorum in Barbaros, hrsg. v. Walter Schätzel (Tübingen 1952). Zur conquista der Neuen Welt als eigentumsrechtliches Problem Deckers, $226 \mathrm{ff}$.

56 Zur Kolonialgesetzgebung Antonio Pérez Martin, in: Coing, Bd. II 2, $242 \mathrm{ff}$.

57 Vázquez, lib. I, cap. 29, nu. 3 mit Nachweisen. 
Vertrauensethik 58 war der Schutz des Eigentums gegen hoheitliche Eingriffe von entscheidender Bedeutung. Dabei mußte das Eigentum, ganz ähnlich dem heutigen verfassungsrechtlichen Begriff, nicht nur im zivilrechtlichen Sinne verstanden werden, sondern als zu schützende individuelle Rechtsposition schlechthin $^{59}$. Zugleich aber mußte, wegen des beginnenden Überseehandels, am Meer die Möglichkeit von Eigentum gerade geleugnet werden. Für die Eigentumsfreiheit mußte das dominium einen naturrechtlichen Status erhalten, der nur bei einer sehr schöpferischen Weiterentwicklung theologischer Erwägungen zum omnia communia einerseits und zu dessen Ausserkraftsetzung andererseits möglich war. Der schwierigste Spagat bestand darin, für das Meer ganz im Gegensatz dazu das omnia communia sogar aufzuwerten. Beide Argumentationen lassen sich - wie zu sehen war - tatsächlich in der iberischen Spätscholastik finden.

\section{Fazit}

Naturrechtliche Überlegungen im Salamanca des 16. Jahrhunderts hatten also offensichtlich sehr säkulare praktische Hintergründe, bedienten sich aber andererseits bekannter Argumentationsmuster aus der theologischen Tradition. Diese Tradition freilich wurde ihrerseits sehr frei und eigenwillig unter Berücksichtigung auch der legistischen und kanonistischen Tradition weiterentwickelt, so wie diesen juristischen Traditionen gegenüber gerade auch bei einem juristischen Aucor die Theologie einen erweiterten Handlungsspielraum bot. Die "Säkularisierung" des Naturrechts erweist sich so als ungemein komplexer Prozeß, den man ebenso gut unter dem Aspekt einer Theologisierung des Rechts erfassen könnte.

58 Bergfeld, 1022; Malte Diesselhorst, Die Lehre des Hugo Grotius vom Versprechen (Köln, Graz 1959) 4 ff.

59 Zur Weite des Begriffs Paolo Grossi, La proprietà nel sistema della seconda scolastica, in: La seconda scolastica nella formazione del diritto privato moderno. Incontro di studi Firenze 16-19 ottobre 1972 (Milano 1973) 117 ff., 124. 
